\title{
RECENSIÓN
}

\section{LUCÍA MARTÍNEZ FERNÁNDEZ}

\author{
El Gravetiense en el sector occidental cantábrico y sus conexiones pirenaicas. \\ Tesis doctoral, Universidad de Oviedo, Facultad de Filosofía y Letras, \\ Departamento de Historia, 2 vol., 1365 p.
}

Ces dernières années ont été particulièrement fécondes en matière de recherche sur le Gravettien de la Péninsule ibérique. Le point d'orgue en a été le colloque d'Altamira en 2011, dont les actes, rapidement publiés et faciles d'accès (Heras et al. 2012), présentent une synthèse complète, suivi des thèses de Paloma de la Peña (2011), de João Marreiros (2013), et de M. Bradtmöller (2013), ainsi que des publications monographiques sur la grotte d'Aitzbitarte III (Altuna et al. 2011) et sur l'atelier plein air de Mugarduia (Barandiarán et al. 2013).

La récente thèse de Lucía Martínez Fernández participe de cette dynamique. Elle aborde, d'une manière générale, le Gravettien de la Péninsule ibérique avec son prolongement pyrénéen, en privilégiant une démarche régionaliste centrée sur la corniche Cantabrique occidentale, approche indispensable et, au bout du compte, la meilleure possible pour progresser dans nos connaissances sur les cultures de la Préhistoire, et son travail de recherche en est une excellente illustration.

Le mémoire se décline principalement en trois parties. La première est consacrée à un état de la question du Gravettien dans l'aire cantabrique et sur le versant sud des Pyrénées occidentales. L'auteure nous donne à lire un vrai travail de (pré)historien, avec son cadre critique des sources archéologiques: documentation exhaustive sur les sites, mais surtout une analyse sans concession des stratigraphies, des séries archéologiques qui en sont issues et une évaluation de leur intégrité. Elle est complétée par un essai de synthèse sur la paléoclimatologie de cette période à partir des données disponibles sur les sites du nord de l'Espagne (sédimentologie, palynologie et faune et micro-faune), exercice difficile compte tenu de la grande disparité de l'information et de ses trop nombreuses lacunes, un vrai casse-tête que peu de spécialiste affronte.

La deuxième partie porte sur l'analyse détaillée de l'industrie lithique gravettienne de trois sites asturiens de référence:

- La Viña, abri orné fouillé entre 1980 et 1996 par le regretté Pr. Javier Fortea (1946-2009: cf. l'ouvrage hommage, sous la direction de Rasilla Vives 2013); ce vaste abri présente une stratigraphie très dilatée, du Moustérien au Magdalénien, comprenant quatre ensembles sédimentaires gravettiens dans le secteur occidental;

- Llonín, grotte ornée recélant un remplissage s'échelonnant du Moustérien au Magdalénien, y com- pris un ensemble stratigraphique du Gravettien final; cette grotte a fait l'objet de nouvelles recherches de 1984 à 2002 sous la direction conjointe de F. Fortea, M. de la Rasilla Vives et V. Rodríguez Otero;

- Cueto de la Mina, abri repris de 1981 à 1986 par M. de la Rasilla Vives, qui a réactualisé les données fournies en 1914 et 1915 par le comte Vega de Sella, notamment par la caractérisation d'un ensemble stratigraphique gravettien.

La démarche de L. Martínez Fernández est tournée vers la caractérisation de l'économie des industries lithiques dans toutes ses composantes: matières premières, techniques et procédés de taille, et structuration de l'outillage. Pour cela, elle a employé la typologique bordienne classique et une méthode novatrice en matière d'étude du débitage, développée ces dernières années à l'université d'Oviedo, notamment par les travaux de David Santamaría (2012). Pour faire court, il s'agit d'une approche d'inspiration laplacienne (cf. travaux de R. Mora et W. Andrefsky) qui se traduit par la constitution d'une grille classificatoire des produits de débitage. Sans chercher à relancer un ancien débat, déplacé cette fois-ci entre technologues «école française " et technologues " courant laplacien », on remarquera toutefois que l'usage d'une terminologie et d'abréviations compliquées peut dérouter le lecteur peu familiarisé à la méthode. Si l'on perçoit son grand avantage, notamment la possibilité de quantifier les données et d'en assurer leur traitement statistique, il lui manque encore, d'une part, la visualisation schématique de tous les éléments définis dans la grille classificatoire, et d'autre part, leur traduction technologique, autrement dit leur place respective au sein des processus de la taille et des chaînes opératoires.

Quoi qu'il en soit, le travail d'acquisition des données, leur traitement et leur analyse ont été un tour de force, compte tenu de la masse d'informations mobilisées. Et les résultats sont au rendez-vous. Pour l'aire cantabrique occidentale, on retiendra principalement les caractéristiques suivantes:

- l'économie des matières premières est tournée essentiellement vers une exploitation locale (silex et quartzite), les silex allochtones provenant de l'est de la chaîne cantabrique sont peu présents dans l'industrie. En fonction du contexte des sources d'approvisionnements et de la situation des sites, des différences appa-

(1) Direction : Pr. Marco de la Rasilla Vives. Soutenue le 23 juillet 2015. 
raissent dans les industries lithiques, tant au niveau des procédés de taille (importance relative des productions d'éclat sur quartzite par exemple) que de la structuration de l'outillage (variabilité des pourcentages des principaux types d'outils: burins de Noailles, lamelles à dos, pointes de la Gravette, grattoirs, burins, pièces esquillées, etc...). Ces variations ne semblent pas être significatives d'une diachronie mais plutôt à mettre en relation avec la nature des sites (occupation de longue durée, ateliers de taille, occupation temporaire axée sur des activités cynégétiques etc.). Toutefois on note quelques tendances: appauvrissement en Burins de Noailles et présence plus affirmée des lamelles à dos au fur et à mesure que l'on progresse vers le Gravettien récent.

II n'y a aucune fléchette ni burin de Raysse, quelques très rares pointes de la Font Robert et pointes à cran... II existe donc une relative stabilité typo-technologique dans les assemblages lithiques et leur évolution diachronique est encore mal perçue du fait de cette stabilité;

- l'industrie osseuse est peu développée, tout comme la parure et l'art mobilier si on compare avec le contexte de l'aire cantabrique orientale et de la zone pyrénéenne. A la Viña, par exemple, on dénombre à peine quelques fragments de poinçon, de sagaies, de dents ou de coquillages perforés (étude en cours). En ce qui concerne les sagaies de type Isturitz, une étude récente en a comptabilisé seulement quatre exemplaires et provenant de sites situés à l'est de la chaîne cantabrique (Rios-Garaizar et Garate 2014). Traditions culturelles différentes, problèmes taphonomiques, ressources animales différentes ou état de la recherche en devenir ? Une réponse ne peut être encore donnée puisqu'il faut attendre les résultats complets de cette thématique de recherche et leur confrontation avec les études archéozoologiques.

La troisième et dernière partie constitue la synthèse de l'ensemble des données et leur intégration dans le panorama du Gravettien de la Péninsule ibérique. Elle est présentée par grands ensembles régionaux qui paraissent pertinents, tout en montrant les relations entre les groupes de la corniche Cantabrique et ceux des Pyrénées centrales, notamment par la mise en évidence d'une grande parenté dans l'économie des industries lithiques (cf. Foucher in Heras et al. 2012; Simonet 2010). Toutefois des différences subsistent avec le versant nord des Pyrénées, où l'industrie osseuse et l'art mobilier y sont beaucoup plus développés (cf. San Juan-Foucher, Aguirre, Goutas et Lacarrière, in Heras et al. 2012; Rivero et Garate 2014). On soulignera ici la nécessité de considérer à part les sites d'Isturitz et de Brassempouy, pour plusieurs raisons. Leur importante fréquentation au Gravettien leur a conféré un statut de site d'agrégation sans conteste, mais la maîtrise de la richesse documentaire rendue difficile par les techniques anciennes de fouille et les mélanges inter-couches avérés ne permettent pas encore de déterminer l'origine précise des groupes, encore moins leur fréquence ou leur dynamique d'occupation. On pressent toutefois le passage de groupes plus septentrionaux (Périgord, voire au-delà): la production des statuettes féminines à Brassempouy, complètement absente au sud des Pyrénées, renvoie nécessairement à des influences nord-orientales européennes.

Les perspectives ouvertes par ce travail de recherche sont nombreuses. L'intégration des données sur les activités symboliques des gravettiens au travers de l'art pariétal est un de ses points forts. La corniche Cantabrique a acquis une telle richesse documentaire au cours de ces 30 dernières années qu'il est désormais possible, dès le Gravettien, d'y concevoir une paléogéographie humaine; ceci dans le sens d'un espace naturel investi par les gravettiens et que l'on peut caractériser à l'aune des critères, économiques, culturels et symboliques.

Enfin, bien que le thème des transitions soit peu développé dans la thèse, les données typo-technologique présentées suggèrent que le niveau $X$ de la Viña (à la base de l'ensemble gravettien) pourrait se rapporter au Gravettien ancien dans une filiation évolutive à partir de l'Aurignacien évolué (le niveau sous-jacent - XI). En particulier si l'on tient compte de certains traits typo-technologiques caractéristiques, comme le débitage selon les modalités qui conduisent à la formation des nucléus de type "grattoirs carénés », de la présence de burins busqués, de lamelles Dufour, associé à une composante gravettienne (lamelles à dos et pointes de la Gravette). Mais il manque encore un cadre radiochronologique développé et une étude comparative très étroite entre ce niveau et le XI sous-jacent. Des mélanges inter-couches sont toujours possibles, bien que l'étude taphonomique entreprise ait déterminé une relativement bonne intégrité des niveaux archéologiques, mais cette supposition n'expliquerait pas à elle seule le fait que le débitage de type « grattoirs carénés » soit présent dans tous les niveaux gravettiens (VIII à X).

Quant à la transition du Gravettien au Solutréen, encore peu connue dans l'aire cantabrique, elle pourrait s'envisager à La Viña (Asturies), à Aitzbitarte III et à Amalda (Pays basque), dans une continuité chronologique et sur les mêmes territoires, en suivant peut-être des mécanismes évolutifs proches de ceux décrits au Portugal (cf. les travaux de Zilhão, Aubry, Almeida, Bicho et Klaric). Hypothèse qui demanderait à être étayée par des travaux de recherche encore en cours et dont les résultats doivent compléter les éléments actuellement manquants du registre (notamment la zone centrale de la Viña).

La thèse de L. Martínez Fernández constitue la somme d'un travail non seulement considérable mais aussi très abouti, et contribue à mieux analyser les phénomènes de régionalisation intervenant au Gravettien sur une échelle européenne. Socle documentaire solide et, on l'espère, point de départ d'une trajectoire d'investigation, elle sera lue avec profit par tout chercheur spécialiste de cette période.

Pascal FOUCHER 


\section{BIBLIOGRAPHIE}

\section{ALTUNA J., MARIEZKURRENA K., RÍOS J.}

2011 Occupaciones humanas en Aitzbitarte III (País Vasco) 33600 - 18400 BP (zona de entrada a la cueva). Vitoria-Gasteiz: Servicio Central de Publicaciones del Gobierno Vasco (EKOB, 5), $536 \mathrm{p}$.

BARANDIARÁN I., CAVA A., AGUIRRE M.

2013 El taller de sílex de Mugarduia Sur. Una ocupación de Urbasa (Navarra) durante el Gravetiense. Vitoria-Gasteiz: Universidad del País Vasco (Veleia. Anejos. Series Maior, 13), $690 \mathrm{p}$.

\section{BRADTMÖLLER M}

2013 Muster jungpaläolithischer Höhlennutzung am Beispiel des Gravettien Nordspaniens. PhD Thesis. Universität zu Köln.

HERAS C. de las, LASHERAS J.-A., A. ARRIZABALAGA A., RASILLA M. de la, dir.

2012 Pensando el Gravetiense: nuevos datos para la región cantábrica en su contexto peninsular y pirenaico, Actas del coloquio de Altamira, 20-22 de octubre 2011 (Monografías del Museo Nacional y Centro de Investigación de Altamira, 23), 619 p. https://sede.educacion. gob.es/publiventa/detalle. action?cod=14457C

\section{MARREIROS J}

2013 Organização e variabilidade das indústrias líticas durante o Gravetense no sudoeste peninsular (Unpublished doctoral dissertation). Universidade do Algarve, Faro.
PEÑA P. de la

2011 Sobre la Unidad tecnológica del Gravetiense en la península ibérica: Implicaciones para el conocimiento del Paleolítico superior inicial. Tesis doctoral. Universidad Complutense de Madrid.

RASILLA VIVES M. de la, dir.

2013 F. Javier Fortea Pérez. Universitatis Ovetensis Magister Estudios en homenaje. Ediciones de la Universidad de Oviedo y Ménsula Ediciones, 594 p.

RÍOS-GARAIZAR J., GARATE D.

2014 Actualisation de l'inventaire des pointes de type Isturitz de la région cantabrique. Paléo, 25, p. 233-245.

RIVERO O., GARATE D.

2014 L'art mobilier gravettien sur support lithique de la grotte d'Isturitz (Saint-Martin d'Arberoue, Pyrénées atlantiques, France): une collection redécouverte. Paléo, 25, p. $247-276$

\section{SANTAMARÍA D.}

2012 La transición del Paleolítico medio al superior en Asturias. El abrigo de la Viña (La Manzaneda, Oviedo) y la cueva de El Sidrón (Borines, Piloña). Tesis doctoral. Universidad de Oviedo.

SIMONET A.

2009 Les gravettiens des Pyrénées. Des armes aux sociétés. Thèse de l'Université de Toulouse-le-Mirail. 